\title{
Thermodynamics of superconductivity beyond the Gorkov decoupling
}

\author{
Shun-ichiro Koh \\ Physics Division, Faculty of Education, Kochi University, Akebono-cho, 2-5-1, Kochi, 780, Japan
}

(Received 12 July 1993; revised manuscript received 13 September 1993)

\begin{abstract}
Superconductivity beyond the Gorkov decoupling is examined with an emphasis on the thermodynamic properties. In the Gorkov decoupling, the interaction term is factorized into the pairing correlation. Therefore, in contrast to the strong pair-pair correlation in coordinate space, the correlation between the Cooper pairs in momentum space is neglected. In this paper, in addition to the conventional pairing interaction, we consider a more general type of attractive interaction that cannot be factorized into the product of the pairing correlation. Accordingly, it becomes necessary to include the neglected correlation. We assume a variational functional where the general type of interaction is taken into account, and determine the state self-consistently at finite temperature. The following results are obtained: (1) The state obtained is more stable at low temperature, compared with the BCS state with the same parameters. (2) As the temperature is increased, our state approaches the BCS state. (3) At the same temperature as in the BCS theory, our system undergoes a phase transition from the superconducting phase to the normal phase.
\end{abstract}

\section{INTRODUCTION}

Gorkov decoupling plays a central role in the selfconsistency mechanism in BCS theory. ${ }^{1}$ The fact that the Gorkov decoupling is a good approximation shows that the Cooper pairs can be considered to behave freely from other pairs in momentum space. ${ }^{2}$ However, because of the increase in the variety of superconducting materials, it is worthwhile reconsidering the validity of this approximation.

One type of superconducting material is the heavyfermion system. ${ }^{3}$ As is well known, the electrons in the normal phase of this system are highly correlated. Nevertheless, the heavy-fermion system becomes a superconductor at low temperature. Thus, there is the possibility that the Cooper pairs formed by these correlated electrons do not behave freely from other pairs, but are correlated as in the normal phase. If this is true, we need to look beyond the Gorkov decoupling to describe them.

Another type of material to consider is the copper oxide high- $T_{c}$ superconductors. Experimental results, such as the appearance of a 2-electron jump both in the Josephson effect ${ }^{4}$ and in flux quantization, ${ }^{5}$ along with the short coherence length, indicate the basic feature of this type of superconductivity. These results suggest that the two electrons in the high- $T_{c}$ superconductors are bound more tightly than in conventional superconductors. However, it is worthwhile considering whether this strong attractive force is described fully by the Gorkov decoupling. If there is a correlation between the Cooper pairs in momentum space, then we need to look beyond the Gorkov decoupling.

In the Gorkov decoupling approximation, the attractive force in the Hamiltonian

$$
H=\sum_{\mathbf{k}, \sigma} \varepsilon_{\mathbf{k}} a_{\mathbf{k} \sigma}^{\dagger} a_{\mathbf{k}, \sigma}+U \sum_{\mathbf{k}, \mathbf{k}^{\prime}, \sigma} a_{\mathbf{k} \sigma}^{\dagger} a_{-\mathbf{k},-\sigma}^{\dagger} a_{-\mathbf{k}^{\prime},-\sigma} a_{\mathbf{k}^{\prime}, \sigma}
$$

is described in terms of the pair correlation; higher-order decouplings such as $\left\langle a_{\mathbf{k} \sigma}^{\dagger} a_{-\mathbf{k},-\sigma^{\dagger}}^{\dagger} a_{-\mathbf{k}^{\prime},-\sigma} a_{\mathbf{k}^{\prime}, \sigma}\right\rangle$ are taken into account by factorizing them into the products of pair correlation, such as $\left\langle a_{\mathbf{k} \sigma}^{\dagger} a_{-\mathbf{k},-\sigma}^{\dagger}\right\rangle$. (This is equivalent to the fact that the BCS ground state is written as the product of different momentum components.) The meaning of this approximation is exemplified by the correlation function for four particles, $f\left(r_{1}, r_{2}, r_{3}, r_{4}\right)$. By the Gorkov decoupling, we pick up only the following type of correlation:

Type A:

$$
f\left(r_{12}\right) f\left(r_{34}\right)+f\left(r_{13}\right) f\left(r_{24}\right)+f\left(r_{14}\right) f\left(r_{23}\right),
$$

where the correlation between particle 1 and 2, for example, is an independent process from that between 3 and 4 . Within this approximation, the Cooper pairs behave freely from other pairs. (There is a strong pair-pair correlation in coordinate space, because of the overlap of the wave functions. However, what is dealt with in this paper is the correlation between the pairs in momentum space. It is unaffected by the extent of the overlap of the wave functions.)

From a theoretical point of view, the attractive force is not restricted to the pairing force. Hence it is not always written in the form of a type-A correlation. As $U$ is increased, another type of correlation for the four particles, type $B$, becomes possible:

Type B:

$$
f\left(r_{12}\right) f\left(r_{13}\right) f\left(r_{14}\right) f\left(r_{23}\right) f\left(r_{24}\right) f\left(r_{34}\right),
$$

in addition to type-A correlation. ${ }^{6}$ In this correlation, approach of the particles 1 and 2 does not prevent particle 3 from approaching particle 1 or 2 . Thus the Cooper pairs cannot be considered to behave freely from other pairs even in momentum space. To formulate type- $A$ and -B correlation consistently, it is insufficient to factorize the higher-order decoupling into the pair correlation. 
Rather, a new self-consistent scheme must be proposed. ${ }^{7}$

Some attempts have been made along this line. Giovannini extended the Gorkov equation so as to include the higher-order decoupling term. ${ }^{8}$ By solving a series of coupled equations, he obtained a result compatible with BCS theory. As somewhat different approach, Gyorffy, Staunton, and Stocks used the coherentpotential approximation (CPA) method. ${ }^{9}$ In this method, the correlation between the Cooper pairs was treated statistically.

Recently, another approach was proposed by Koh. ${ }^{10,11}$ In this approach, an imaginary mean-field $\Gamma(\omega)$ that interacts with the Cooper pairs was introduced, in order to study the pairing due to the attractive type-B interaction. As a result, the Cooper pairs interact with each other in momentum space. The properties of the mean field $\Gamma(\omega)$ must be determined self-consistently by the electron system.

To formulate this idea, a thermodynamic potential $\Omega$ that consisted of the order parameter $\phi(\omega)$ and the mean filed $\Gamma(\omega)$ was assumed. By making use of the fact that $\Omega$ is stationary with respect to variations in $\phi(\omega)$ and $\Gamma(\omega)$, the equilibrium state is determined selfconsistently. ${ }^{15}$ Such a functional consists of the following three parts:

(1) The potential that yields BCS theory, derived by Eliashberg as follows, ${ }^{16}$

$$
\begin{aligned}
\Omega_{1}= & -\frac{1}{\beta} \sum_{p} \ln [-\psi(p)]+\frac{2}{\beta} \sum_{p} \phi^{*}(p) F(p) \\
& +U\left[\frac{1}{\beta}\right]^{2} \sum_{p} \sum_{p^{\prime}} F^{*}(p) F\left(p^{\prime}\right),
\end{aligned}
$$

where the third term comes from the Gorkov decoupling (see Appendix A).

(2) An extra energy that exists because of the correlation between the pairs in momentum space:

$$
\Omega_{2}=\frac{1}{U^{2}} \frac{1}{\beta} \sum_{i \varepsilon_{n}} \Gamma\left(i \varepsilon_{n}\right) \Gamma\left(-i \varepsilon_{n}\right) .
$$

This energy is assumed to be of the field-energy form of $\Gamma(\omega)$. This is always positive, and therefore necessary for the stability of the system.

(3) A term that expresses the interaction between the mean field and the Cooper pairs,

$$
\Omega_{3}=\left(\frac{1}{\beta}\right)^{2} \sum_{p} \sum_{p^{\prime}} F^{*}(p) \Gamma\left(i \omega_{n}-i \omega_{n^{\prime}}\right) F\left(p^{\prime}\right),
$$

where an anomalous Green function $F$ is defined as follows,

$$
\begin{aligned}
& F(p)=\frac{-\phi\left(i \omega_{n}\right)}{\psi(p)}, \\
& \psi(p)=\left(i \omega_{n}\right)^{2}-\varepsilon_{\mathbf{k}}^{2}-\left|\phi\left(i \omega_{n}\right)\right|^{2},
\end{aligned}
$$

and $p=\left(\mathbf{k}, i \omega_{n}\right)$ for fermions and $q=\left(\mathbf{k}, i \varepsilon_{n}\right)$ for bosons. ${ }^{17}$ Here $\Omega_{3}$ is obtained by replacing $U$ with $\Gamma(\omega)$ in the third term of the right-hand side of $\Omega_{1}$. It is possible to assume other forms that include more mean fields
$\Gamma(\omega)$ and more anomalous Green functions $F$ than in Eq. (3). However, in a system slightly modified from the BCS system, these terms can be considered as higher-order terms. For small $\Gamma$, the magnitude of Eq. (3) is far greater than that of the higher-order terms. Thus, it is appropriate to take only the lowest-order term, Eq. (3), as a first approximation.

The thermodynamic potential $\Omega_{1}+\Omega_{2}+\Omega_{3}$ has the following meaning. The starting point of Refs. 10 and 11 is the same Hamiltonian as in the BCS theory. Thus the introduction of $\Omega_{2}+\Omega_{3}$ to the potential is a different approximation for the interaction term. In order to understand the meaning of $\Omega_{2}+\Omega_{3}$, it is useful to compare the present method with the kinematic-equation method in Ref. 2. When we calculate the Gorkov equation one step further than usual, not only four-operator products, but also eight-operator products appear. These products are factorized into two possible forms, that is, two $\Gamma$ 's in $\Omega_{2}$, and one $\Gamma$ and two $F$ 's in $\Omega_{3}$. This is a natural extension of the Gorkov decoupling to the higher-order terms.

$\Omega_{1}+\Omega_{2}+\Omega_{3}$ is stationary with respect to variations in $\phi(\omega), \phi(-\omega), \Gamma(\omega)$, and $\Gamma(-\omega)$, if these quantities are given by,

$$
\begin{aligned}
& \phi\left(i \omega_{n}\right)=-\frac{1}{\beta} \sum_{i \omega_{n}} \sum_{\mathbf{k}^{\prime}}\left[U+\Gamma\left(i \omega_{n}-i \omega_{n^{\prime}}\right)\right] F\left(p^{\prime}\right), \\
& \Gamma\left(i \varepsilon_{n_{2}}\right)=-U^{2} \frac{1}{\beta} \sum_{i \omega_{n_{1}}} \sum_{\mathbf{k}_{1}} \sum_{\mathbf{k}_{2}>0} F^{*}\left(p_{1}+q_{2}\right) F\left(p_{1}\right) .
\end{aligned}
$$

If we let $\Gamma(\omega) \rightarrow 0$ in Eq. (5), it is reduced to the wellknown BCS gap equation. However, a set of coupled integral equations determining $\phi$ and $\Gamma$ must be solved.

The self-consistency mechanism expressed by Eqs. (5) and (6) is illustrated in Fig. 1, where the lines with two opposite arrows represent the anomalous Green function $F$, Eq. (4), and the dotted lines represent $U$. In Eq. (5), $\Gamma$ as well as $U$ appears as the attractive interaction responsible for the pairing. Accordingly, not only Fig. 1(a) but also Fig. 1(b) is necessary. In Fig. 1(b), the anomalous Green functions appear both in the bubble part and in the upper and lower parts. They must be treated equally, and therefore must be determined self-consistently. In this sense, Eqs. (5) and (6) include a mechanism beyond the Gorkov decoupling.

A minimum value of $\Omega$ is obtained by use of Eqs. (1) $-(6)$.

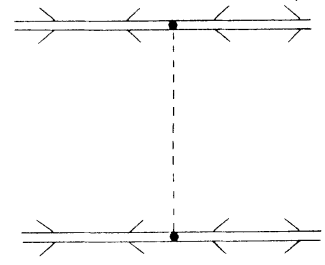

(a)

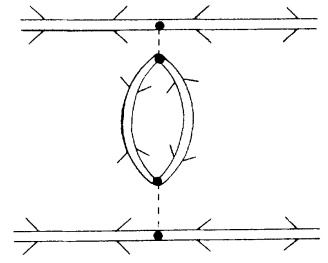

( b )
FIG. 1. Two types of interaction. (a) Interaction in Gorkov decoupling, and (b) interaction added to (a) in the present treatment. The line with two opposite arrows represents the anomalous Green function $F$, and the dotted line the interaction $U$. 


$$
\begin{aligned}
F_{s}= & -\frac{1}{\beta} \sum_{p}\left[\ln [-\psi(p)]+\frac{|\phi(p)|^{2}}{\psi(p)}\right] \\
& +\frac{1}{U^{2}} \frac{1}{\beta} \sum_{i \varepsilon_{n}} \Gamma\left(i \varepsilon_{n}\right) \Gamma\left(-i \varepsilon_{n}\right)
\end{aligned}
$$

The first term corresponds to $F_{s}$ in the BCS theory, whereas the second term comes from the correlation beyond the Gorkov decoupling. The first term is negative, but the second term is always positive. The cancellation of these two terms determines whether or not the superconductivity beyond Gorkov decoupling is more stable than conventional superconductivity.

It is possible to consider the stability of the system from the structure of the thermodynamic potential $\Omega\left(=\Omega_{1}+\Omega_{2}+\Omega_{3}\right): \Omega_{2}$ is always positive, but $\Omega_{1}+\Omega_{3}$ becomes negative when $\Gamma(\omega)$ becomes negative, due to the pairing enhancement by $\Gamma$. As for the magnitude, $\Omega_{2}$ is of second order in $\Gamma$, whereas $\Omega_{3}$ is of first order. Hence, for small negative $\Gamma, \Omega_{3}$ is greater than $\Omega_{2}$, and therefore $\Omega$ becomes lower than $\Omega_{1}$, the BCS potential. Thus the present system becomes more stable than the BCS system. Reference 10 shows a model calculation of this result at zero temperature.

This paper extends the study in Ref. 10 to nonzero temperature. Section II describes a formalism for this extension, while Sec. III presents a model calculation and Sec. IV the results. Section $V$ discusses the physical meaning of the results and their relevance for real materials.

\section{FORMALISM}

The formula in Eqs. (1)-(7) of Sec. I can be rewritten to study the thermodynamic properties of the present system. ${ }^{12-14,18,19}$ For simplicity, we assume the following conditions: (1) isotropy of the system, (2) electron-hole symmetry with a cutoff frequency $\omega_{c}$, and (3) constant density of states $N(0)$.

Transforming the summation in Eqs. (1)-(3) into an integral gives the thermodynamic potential difference between the normal and the superconducting states $\Omega_{s}-\Omega_{n}\left(=\Omega_{1}^{\prime}+\Omega_{2}^{\prime}+\Omega_{3}^{\prime}\right):$

$$
\begin{aligned}
& \Omega_{1}^{\prime}=-2 N(0) \operatorname{Re} \int_{0}^{\omega_{c}} d \omega\left[\omega-\sqrt{\omega^{2}-\phi^{2}(\omega, T)}\right. \\
& \left.-\frac{\phi^{2}(\omega, T)}{\sqrt{\omega^{2}-\phi^{2}(\omega, T)}}\right] \\
& \times \tanh \left(\frac{\beta \omega}{2}\right) \text {, } \\
& \Omega_{2}^{\prime}=\frac{1}{U^{2}} \iint \frac{d \omega d \omega^{\prime}}{\pi^{2}} \operatorname{Im} \Gamma(\omega, T) \operatorname{Im} \Gamma\left(\omega^{\prime}, T\right) \\
& \times \frac{n(\omega)-n\left(-\omega^{\prime}\right)}{\omega+\omega^{\prime}}, \\
& \Omega_{3}^{\prime}=N^{2}(0) \operatorname{Re} \iint \frac{\phi(\omega, T)}{\sqrt{\omega^{2}-\phi^{2}(\omega, T)}} \tanh \left(\frac{\beta \omega}{2}\right) \\
& \times\left[U+\Gamma\left(\omega-\omega^{\prime}, T\right)\right] \frac{\phi\left(\omega^{\prime}, T\right)}{\sqrt{\omega^{\prime 2}-\phi^{2}\left(\omega^{\prime}, T\right)}} \\
& \times \tanh \left(\frac{\beta \omega^{\prime}}{2}\right) d \omega d \omega^{\prime},
\end{aligned}
$$

where $n(\omega)$ is the Bose function. The third term on the right-hand side of $\Omega_{1}$, Eq. (1), is included in $\Omega_{3}^{\prime}$.

Similarly, the self-consistency equation (5) can be rewritten as follows,

$$
\begin{aligned}
\phi(\omega, T)=-N(0) & \int_{-\omega_{c}}^{\omega_{c}} d x[U+\Gamma(\omega-x, T)] \\
& \times \frac{\phi(x, T)}{\sqrt{x^{2}-\phi^{2}(x, T)}} \tanh \left(\frac{\beta x}{2}\right) .
\end{aligned}
$$

Transforming the summation in Eq. (6) into an integral yields

$$
\Gamma(\omega, T)=\frac{U^{2}}{2} \iint g_{+}(\omega, \mathbf{k}, \mathbf{q})\left[f\left(-E_{\mathbf{k}}\right)-f\left(E_{\mathbf{k}+\mathbf{q}}\right)\right] \frac{d \mathbf{k} d \mathbf{q}}{(2 \pi)^{6}}+\frac{U^{2}}{2} \iint g_{-}(\omega, \mathbf{k}, \mathbf{q})\left[f\left(E_{\mathbf{k}}\right)-f\left(E_{\mathbf{k}+\mathbf{q}}\right)\right] \frac{d \mathbf{k} d \mathbf{q}}{(2 \pi)^{6}},
$$

where

$$
\begin{aligned}
& g_{ \pm}(\omega, \mathbf{k}, \mathbf{q})=\frac{\bar{\phi}^{2}}{E_{\mathbf{k}} E_{\mathbf{k}+\mathbf{q}}}\left(\frac{1}{\omega-E_{\mathbf{k}} \mp E_{\mathbf{k}+\mathbf{q}}}-\frac{1}{\omega+E_{\mathbf{k}} \pm E_{\mathbf{k}+\mathbf{q}}}\right), \\
& E_{\mathbf{k}}=\sqrt{\varepsilon_{\mathbf{k}}^{2}+\bar{\phi}^{2}(T)}
\end{aligned}
$$

and $f(\omega)$ is the Fermi function. As in Refs. 10 and 11, the frequency dependence of $\phi(\omega, T)$ is neglected in the righthand side of Eq. (6), and the averaged form $\bar{\phi}(T)$ is used instead of $\phi(\omega, T)$. The coupled integral equations (11)-(13) determine $\phi(\omega, T)$ and $\Gamma(\omega, T)$, where $\bar{\phi}(T)$ is calculated from $\phi(\omega, T) .{ }^{20}$

Equations (11)-(13) illustrate the following properties of the present system.

(a) As the temperature is increased, the ratio of $\Gamma$ to $U$ decreases gradually on the right-handed side of Eq. (11). This means that the present system approaches the BCS system. Finally, at the transition temperature $T_{c}, \Gamma$ vanishes and then the present system is reduced to the BCS system. Consequently, $T_{c}$ of the present system is exactly the same as that of the BCS system.

(b) In Eq. (11), the contribution from the Gorkov decoupling is of order $N(0) U$, while that beyond the Gorkov decou- 
pling must be estimated carefully. The mean-field $\Gamma$ must be of order $N(0)^{2} U^{2} \omega_{c}$, because the anomalous Green function $F$ of order $1 / \omega_{c}$ is summed up to $\omega_{c}$ in Eq. (6). Consequently, the magnitude of the contribution beyond the Gorkov decoupling is of order $N(0)^{3} U^{2} \omega_{c}$ in Eq. (11). Due to this term, the thermodynamic functions of our system cannot be considered as functions of $N(0) U$. Among the different systems that have the same $N(0) U$, a system with high $N(0)$ and weak $U$ shows the tendency to superconductivity beyond the Gorkov decoupling more evidently than the system with low $N(0)$ and strong $U$. This property allows us to estimate the extent to which the present system is modified from the BCS system. Sections III and IV elaborate on this point and give numerical solutions to Eqs. (11)-(13).

By substituting Eqs. (11)-(13) into Eqs. (8) $-(10)$, we find the free-energy difference $F_{s}-F_{n}$ is given by

$$
F_{s}-F_{n}=-2 N(0) \operatorname{Re} \int_{0}^{\omega_{c}} d \omega\left[\omega-\sqrt{\omega^{2}-\phi^{2}(\omega, T)}-\frac{\phi^{2}(\omega, T)}{2 \sqrt{\omega^{2}-\phi^{2}(\omega, T)}}\right] \tanh \left(\frac{\beta \omega}{2}\right)+\Omega_{2}^{\prime} .
$$

Equation (14) can be treated as in Ref. 18 and rewritten with the following steps: (1) Divide Eq. (14) into two parts; one including either $n(\omega)$ or $f(\omega)$, and another including neither. (2) Assume the $\omega$ dependence of $\phi(\omega, T)$ to be weak, as is confirmed in Sec. IV, and change the variable of integration. Thus we find,

$$
\begin{aligned}
F_{s}= & 2 N(0) \int_{0}^{\omega_{c}} d \varepsilon\left[\varepsilon-\sqrt{\varepsilon^{2}+\phi^{2}(\varepsilon, T)}+\frac{\phi^{2}(\varepsilon, T)}{2 \sqrt{\varepsilon^{2}+\phi^{2}(\varepsilon, T)}}\right] \\
& +4 N(0) \int_{0}^{\omega_{c}} d \varepsilon\left[-\sqrt{\varepsilon^{2}+\phi^{2}(\varepsilon, T)}+\frac{\phi^{2}(\varepsilon, T)}{2 \sqrt{\varepsilon^{2}+\phi^{2}(\varepsilon, T)}}\right] f\left(\sqrt{\varepsilon^{2}+\phi^{2}(\varepsilon, T)}\right) d \varepsilon \\
& +\frac{1}{U^{2}} \int_{0}^{2 \omega_{c}} \frac{d \omega}{2 \pi} \Gamma(\omega, T) \Gamma(-\omega, T)+\frac{1}{U^{2}} \iint \frac{d \omega d \omega^{\prime}}{\pi^{2}} \operatorname{Im} \Gamma(\omega, T) \operatorname{Im} \Gamma\left(\omega^{\prime}, T\right) \frac{n(\omega)+n\left(\omega^{\prime}\right)}{\omega+\omega^{\prime}} .
\end{aligned}
$$

To obtain the entropy, it is appropriate to begin with $\Omega$. The solution of Eqs. (11) and (12) satisfies the equations $\partial \Omega / \partial \phi=0$ and $\partial \Omega / \partial \Gamma=0$. Consequently, the entropy $S(=-\partial F / \partial T)$ equals $-\partial \Omega / \partial T$, if Eqs. (11) and (12) are satisfied. By differentiating $\Omega_{s}-\Omega_{n}$ explicitly with respect to $T$ in $\tanh (\beta \omega / 2)$ and $n(\omega)$ rather than implicitly with respect to $T$ in $\phi(\omega, T)$ and $\Gamma(\omega, T)$, the entropy difference is given by

$$
\begin{aligned}
S_{s}-S_{n}= & -\frac{N(0)}{k_{B} T^{2}} \operatorname{Re} \int_{0}^{\omega_{c}} d \omega\left[\omega-\sqrt{\omega^{2}-\phi^{2}(\omega, T)}-\frac{\phi^{2}(\omega, T)}{\sqrt{\omega^{2}-\phi^{2}(\omega, T)}}\right] \omega \operatorname{sech}^{2}\left[\frac{\beta \omega}{2}\right] \\
& +\frac{N^{2}(0)}{2 k_{B} T^{2}} \operatorname{Re}_{0}^{\omega_{c}} \int_{0}^{\omega_{c}} \frac{\phi(\omega, T)}{\sqrt{\omega^{2}-\phi^{2}(\omega, T)}}\left[\omega \operatorname{sech}^{2}\left[\frac{\beta \omega}{2}\right] \tanh \left[\frac{\beta \omega^{\prime}}{2}\right]+\omega^{\prime} \tanh \left[\frac{\beta \omega}{2}\right] \operatorname{sech}^{2}\left[\frac{\beta \omega^{\prime}}{2}\right]\right] \\
& \times\left[U+\Gamma\left(\omega-\omega^{\prime}, T\right)\right] \frac{\phi\left(\omega^{\prime}, T\right)}{\sqrt{\omega^{\prime 2}-\phi^{2}\left(\omega^{\prime}, T\right)}} d \omega d \omega^{\prime}-\frac{\partial \Omega_{2}^{\prime}}{\partial T} .
\end{aligned}
$$

Using Eq. (11), the second term in the right-hand side of Eq. (16) can be simplified. Thus we find,

$$
\begin{aligned}
S_{s}= & \frac{N(0)}{k_{B} T^{2}} \int_{\phi}^{\omega_{c}} \omega \sqrt{\omega^{2}-\phi^{2}(\omega, T)} \operatorname{sech}^{2}\left(\frac{\beta \omega}{2}\right) d \omega \\
& +\frac{1}{4 U^{2} k_{B} T^{2}} \iint \frac{d \omega d \omega^{\prime}}{\pi^{2}} \frac{\operatorname{Im} \Gamma(\omega, T) \operatorname{Im} \Gamma\left(\omega^{\prime}, T\right)}{\omega+\omega^{\prime}}\left[\omega \operatorname{cosech}^{2}\left[\frac{\beta \omega}{2}\right)+\omega^{\prime} \operatorname{cosech}^{2}\left(\frac{\beta \omega^{\prime}}{2}\right)\right],
\end{aligned}
$$

where the first term agrees with the expression derived by Eliashberg.

Finally, using Eqs. (15) and (17), the energy of the present system $E_{s}\left(=F_{s}+T S_{s}\right)$ is obtained.

\section{MODEL CALCULATION}

\section{A. The self-consistency equation}

To carry out the integration in Eq. (12), the variables in it are changed from $\mathbf{k}$ and $\mathbf{k}+\mathbf{q}$ to $\varepsilon$ and $\varepsilon^{\prime}$ respectively, as in Ref. 10. Next, the term including $f\left(E_{\mathbf{k}+\mathbf{q}}\right)$ is integrated over $\varepsilon$, and the term including $f\left(E_{\mathbf{k}}\right)$ over $\varepsilon^{\prime}$ (for details, see Appendix B). Thus we find,

$$
\begin{aligned}
\Gamma(\omega, T)= & -\frac{(N U)^{2}}{2} \int_{-\omega_{c}}^{0} d \varepsilon[M(\varepsilon, \omega)+M(\varepsilon,-\omega)]\left[1-2 f\left(\sqrt{\varepsilon^{2}+\bar{\phi}^{2}(T)}\right)\right] \\
& +\frac{(N U)^{2}}{2} \int_{-\omega_{c}}^{0} d \varepsilon\left[L(\varepsilon, \omega)+L(\varepsilon,-\omega)+M^{\prime}(\varepsilon, \omega)+M^{\prime}(\varepsilon,-\omega)\right] f\left(\sqrt{\varepsilon^{2}+\vec{\phi}^{2}(T)}\right),
\end{aligned}
$$


where

$$
\begin{aligned}
& M(\varepsilon, \omega)=\frac{\bar{\phi}^{2}(T)}{\sqrt{\varepsilon^{2}+\bar{\phi}^{2}(T)}}\left[I_{s}^{(1)}(\varepsilon, \omega)+\widetilde{\omega} I_{s}^{(2)}(\varepsilon, \omega)\right], \\
& L(\varepsilon, \omega)=\frac{\bar{\phi}^{2}(T)}{\sqrt{\varepsilon^{2}+\bar{\phi}^{2}(T)}}\left[I_{s}^{(1)}(\varepsilon, \omega)-\widetilde{\omega} I_{s}^{(2)}(\varepsilon, \omega)\right], \\
& M^{\prime}(\varepsilon, \omega)=\frac{\bar{\phi}^{2}(T)}{\sqrt{\varepsilon^{2}+\bar{\phi}^{2}(T)}}\left[I_{s}^{(1)}(\varepsilon, \omega)+\widetilde{\omega}^{\prime} I_{s}^{\prime(2)}(\varepsilon, \omega)\right] .
\end{aligned}
$$

The functions $I_{s}^{(1)}$ and $I_{s}^{(2)}$ in Eq. (19) are defined as follows,

$$
\begin{aligned}
& I_{s}^{(1)}(\varepsilon, \omega)=\frac{1}{\sqrt{\left|\bar{\phi}^{2}(T)-\widetilde{\omega}^{2}\right|}}\left\{\begin{array}{l}
\tan ^{-1}\left(\frac{\omega_{c}}{\sqrt{\bar{\phi}^{2}(T)-\widetilde{\omega}^{2}}}\right), \\
\frac{1}{2} \ln \left|\frac{\omega_{c}-\sqrt{\widetilde{\omega}^{2}-\bar{\phi}^{2}(T)}}{\omega_{c}+\sqrt{\widetilde{\omega}^{2}-\bar{\phi}^{2}(T)}}\right|, \quad \bar{\phi}^{2}(T)<\widetilde{\omega}^{2},
\end{array}\right.
\end{aligned}
$$

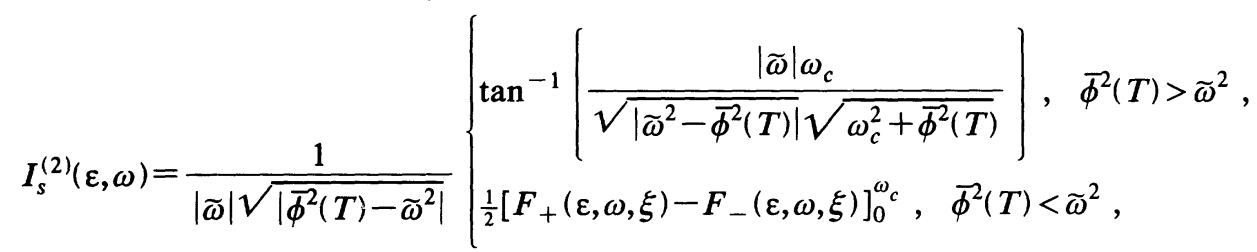

$$
\begin{aligned}
& F_{ \pm}(\varepsilon, \omega, \xi)=\ln \left| \pm \sqrt{\widetilde{\omega}^{2}-\bar{\phi}^{2}(T)}+\frac{|\widetilde{\omega}|-\sqrt{\xi^{2}+\bar{\phi}^{2}(T)}}{\xi \mp \sqrt{\widetilde{\omega}^{2}-\bar{\phi}^{2}(T)}}\right| \widetilde{\omega}||,
\end{aligned}
$$

and $\widetilde{\omega}=\omega-\sqrt{\varepsilon^{2}+\bar{\phi}^{2}} \cdot I_{s}^{(1)}$ and $I_{s}^{\prime(2)}$ are the same functions as $I_{s}^{(1)}$ and $I_{s}^{(2)}$, respectively, except that $\widetilde{\omega}$ in $I_{s}^{(1)}$ and $I_{s}^{(2)}$ is replaced by $\widetilde{\omega}^{\prime}=\omega+\sqrt{\varepsilon^{2}+\bar{\phi}^{2}}$.

Using Eqs. (11) and (18), the solution $\phi(\omega, T)$ and $\Gamma(\omega, T)$ is obtained numerically by iteration.

\section{B. The free energy and the entropy}

$\operatorname{Im} \Gamma(\omega, T)$ in Eqs. (15) and (17) is derived from Eqs. (12) and (13) as follows,

$\operatorname{Im} \Gamma(\omega, T)=\frac{(N U)^{2}}{2} \pi \iint I_{a}\left(\omega, \varepsilon, \varepsilon^{\prime}\right)\left[f(-E)-f\left(E^{\prime}\right)\right] d \varepsilon d \varepsilon^{\prime}+\frac{(N U)^{2}}{2} \pi \iint I_{b}\left(\omega, \varepsilon, \varepsilon^{\prime}\right)\left[f(E)-f\left(E^{\prime}\right)\right] d \varepsilon d \varepsilon^{\prime}$,

$I_{a, b}\left(\omega, \varepsilon, \varepsilon^{\prime}\right)=\frac{\bar{\phi}^{2}}{E E^{\prime}}\left[\delta\left(\omega-E \mp E^{\prime}\right)-\delta\left(\omega+E \pm E^{\prime}\right)\right]$.

Using the solution $\phi(\omega, T)$ and $\Gamma(\omega, T)$, the numerical integration over $\varepsilon$ and $\varepsilon^{\prime}$ is carried out with random variables. The result is shown in Sec. IV.

\section{RESULTS}

\section{A. The order parameter $\phi(\omega, T)$, and the mean field $\Gamma(\omega, T)$}

In Fig. 2, the self-consistent solution $\phi(\omega, T)$ and $\Gamma(\omega, T)$ is plotted as a solid curve at $T / T_{c}=0$ in (a), $T / T_{c}=0.4$ in (b), $T / T_{c}=0.9$ in (c), and $T / T_{c}=0.95$ in (d). The curves are calculated using Eqs. (11)-(13), with $\omega_{c}=10 \mathrm{meV}, U=-50 \mathrm{meV}$, and $N(0)=0.01 / \mathrm{meV}$. The BCS prediction of the order parameter is shown as the dotted line. The order parameter, $\phi(\omega, T)$ is enhanced by nonzero $\Gamma(\omega, T)$ from the BCS prediction. As the temperature is increased, however, $\Gamma(\omega, T)$ tends to zero. Correspondingly, $\phi(\omega, T)$ approaches the BCS prediction.

\section{B. Temperature dependence of the order parameter}

In Fig. 3, the frequency-averaged order parameter $\bar{\phi}(T)$ is plotted as a thick solid curve. $\bar{\phi}(T)$ is obtained by the same calculation as in Fig. 2. The BCS prediction $\phi_{\mathrm{BCS}}(T)$ is shown as a thin solid curve. Although $\bar{\phi}(T)$ is enhanced from $\phi_{\mathrm{BCS}}(T)$, both quantities tend to zero at the same transition temperature. Therefore, the ratio $2 \bar{\phi}(T=0) / k T_{c}$ in the present system is larger than 3.52 , the well-known BCS value.

\section{Temperature dependence of the free energy}

In Fig. 4 , the free energy $F_{s}$ is plotted as a thick solid curve. $F_{s}(T)$ is calculated by Eq. (15), using the same parameters as in Fig. 2. It is compared with the BCS prediction $F_{\mathrm{BCS}}(T)$, a thin solid curve. For reference, the free energy of the normal Fermi gas is given as a thin 

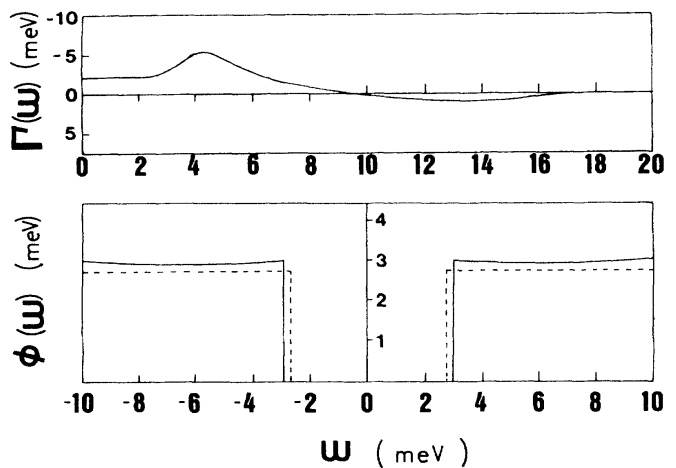

(a)
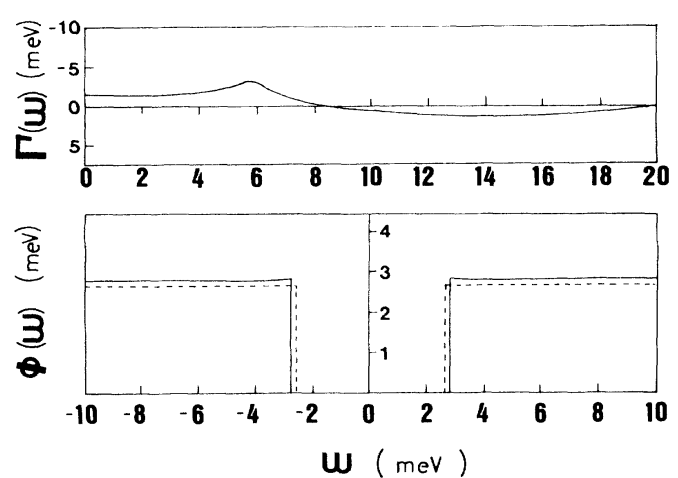

(b)
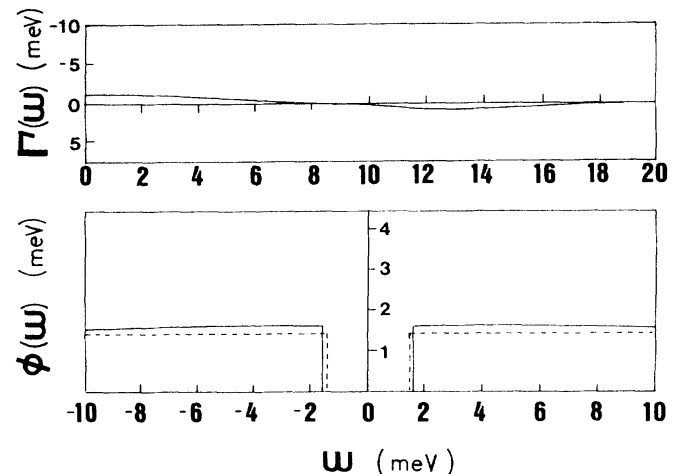

(c)
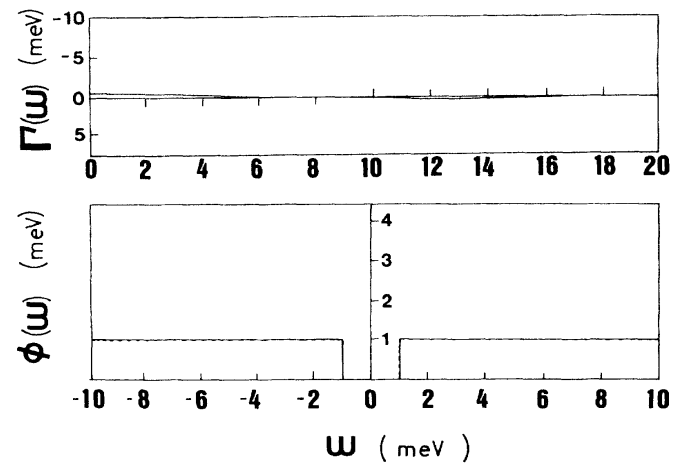

(d)

FIG. 2. Self-consistent solution $\Gamma(\omega, T)$ and $\phi(\omega, T)$. The thick line is at $T / T_{c}=0$ in (a), at $T / T_{c}=0.4$ in (b), at $T / T_{c}=0.9$ in (c), and at $T / T_{c}=0.95$ in (d), for $N(0)=0.01 / \mathrm{meV}, U=-50 \mathrm{meV}$, and $\omega_{c}=10 \mathrm{meV}$. The dotted straight line at the bottom of each figure is the BCS prediction of the order parameter.

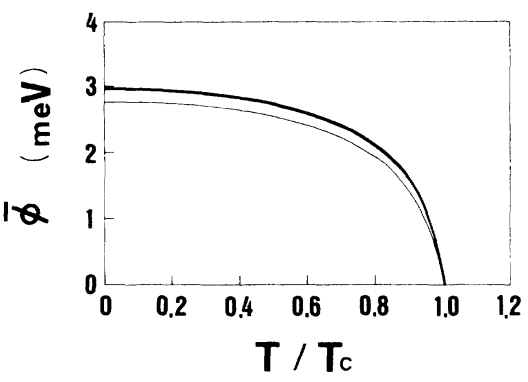

FIG. 3. Temperature dependence of the frequency-averaged order parameter $\bar{\phi}(T)$. The thick solid line gives that of the present model with the same parameters as in Fig. 2. The thin solid line is the BCS prediction.

solid curve that passes through the origin. The present system is more stable than the BCS system. But, as the temperature is increased, $F_{s}-F_{n}$ of the present system approaches the BCS predicted value.

\section{Temperature dependence of the entropy}

In Fig. 5, the entropy $S_{s}(T)$ is plotted as a thick solid curve. It is obtained by Eq. (17) using the same parameters as in Fig. 2. The BCS prediction $S_{\mathrm{BCS}}(T)$ is shown as a thin solid curve. For reference, the entropy of the normal Fermi gas $S_{n}(T)$ is shown as a straight line. $S_{s}(T)$ is always lower than $S_{\mathrm{BCS}}(T)$.

\section{E. Temperature dependence of the energy}

In Fig. 6, the energy $E_{s}(T)$ is plotted as a thick solid curve. $E_{s}(T)$ is calculated by Eqs. (15) and (17) with the same parameters as in Fig. 2. It is compared with the BCS prediction $E_{\mathrm{BCS}}(T)$, a thin solid curve. For reference, the energy of the normal Fermi gas $E_{n}(T)$ is shown

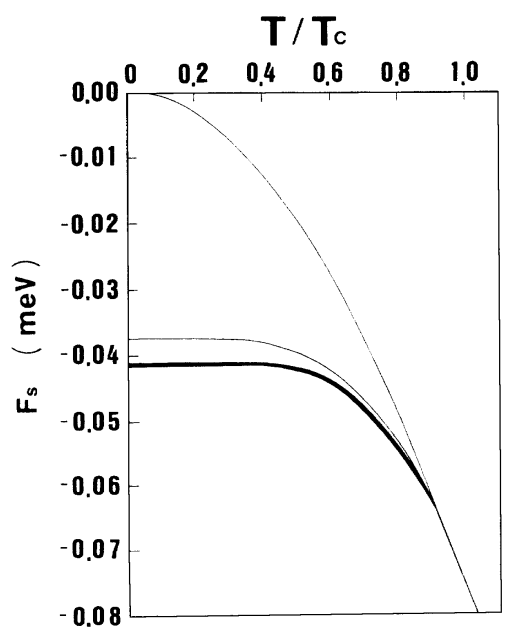

FIG. 4. Temperature dependence of the free energy $F_{s}(T)$. The thick solid line gives that of the present model, calculated using Eq. (15) with the same parameters as in Fig. 2. The thin solid line is the BCS prediction. For reference, the free energy of the normal electron gas $F_{n}(T)$ is depicted as a thin solid line that passes through the origin. 


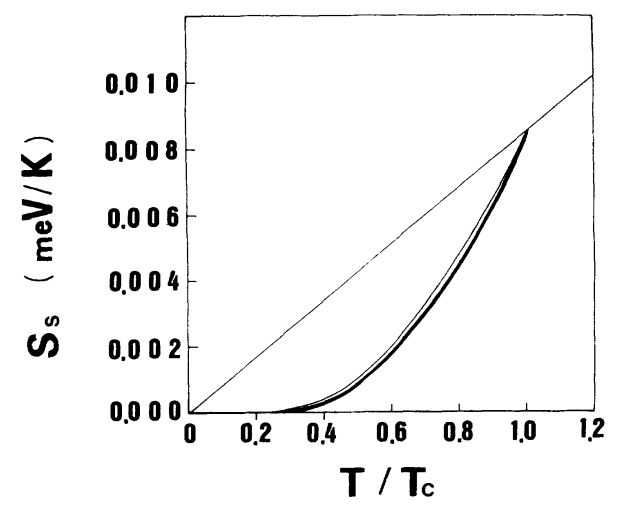

FIG. 5. Temperature dependence of the entropy $S_{s}(T)$. The thick solid line gives that of the present model, calculated using Eq. (17) with the same parameters as in Fig. 2. The thin solid line is the BCS prediction. For reference, the entropy of the normal electron gas $S_{n}(T)$ is depicted as a thin straight line.

as a thin solid curve that passes through the origin. $E_{s}(T)$ is lower than $E_{\mathrm{BCS}}(T)$ at low temperature, but, in the vicinity just below $T_{c}, E_{s}(T)$ approaches $E_{\mathrm{BCS}}(T)$ rapidly.

\section{F. $N(0)$ dependence of the free energy}

In Fig. 7, the free-energy difference $F_{s}-F_{n}$ as a function of $N(0)$ is plotted as a solid curve at $T / T_{c}=0$ in Fig. $7(\mathrm{a})$, and $T / T_{c}=0.4$ in Fig. $7(\mathrm{~b})$. The curves were calculated using Eq. (15) with $N(0) U=-0.5$. [ $U$ is changed accordingly, to maintain constant $N(0) U$.] The prediction of the BCS theory, $-\frac{1}{2} N(0) U^{2}$, is shown as a straight line. Compared with $F_{s}-F_{n}$ of the BCS system, $F_{s}-F_{n}$ of the present system has a strong dependence on $N(0)$ both at $T / T_{c}=0$ and at $T / T_{c}=0.4$. Compared with the result at $T / T_{c}=0$, the $N(0)$ dependence of $F_{s}-F_{n}$ at $T / T_{c}=0.4$ differs only slightly from the BCS prediction.

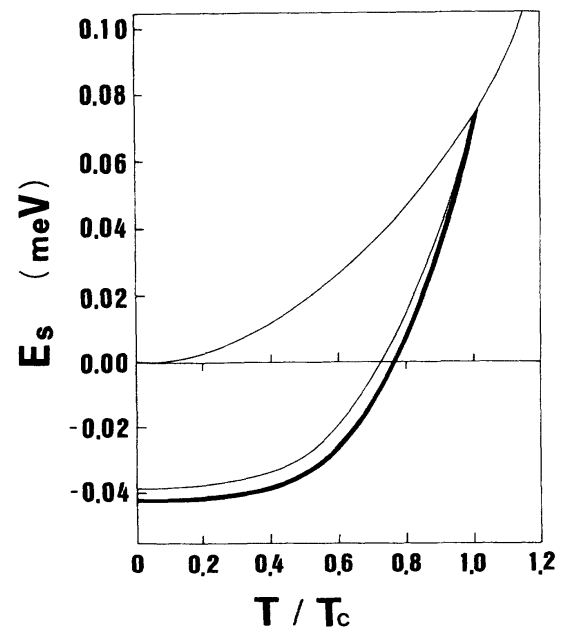

FIG. 6. Temperature dependence of the energy $E_{s}(T)$. The thick solid line gives that of the present model, calculated using Eqs. (15) and (17) with the same parameters as in Fig. 2. The thin solid line is the BCS prediction. For reference, the energy of the normal electron gas $E_{n}(T)$ is depicted as a thin solid line that passes through the origin.

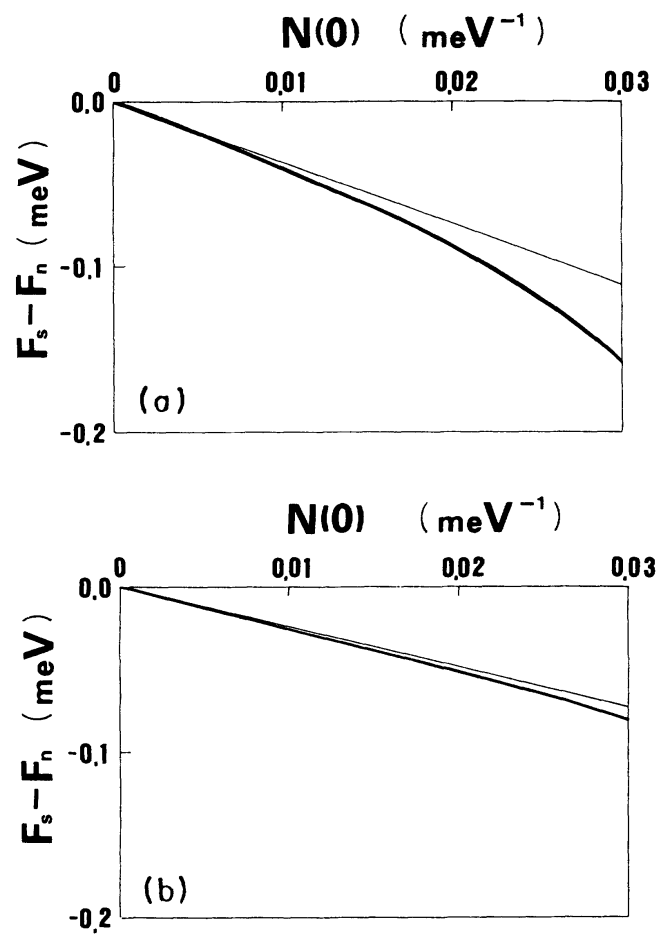

FIG. 7. Temperature change of the $N(0)$ dependence of the free-energy difference $F_{s}-F_{n}$, (a) at $T / T_{c}=0$, and (b) at $T / T_{c}=0.4$, calculated using Eq. (15) with $\omega_{c}=10 \mathrm{meV}$ for constant $N(0) U=-0.5$. The thick solid line gives the $N(0)$ dependence of $F_{s}-F_{n}$ of the present system. The thin straight line is the BCS prediction.

\section{DISCUSSION}

\section{A. Qualitative features of the superconductivity beyond the Gorkov decoupling}

In the BCS theory, the two electrons are bound loosely. The attractive force responsible for this pairing is assumed explicitly in the Hamiltonian. On the other hand, the repulsive force is not explicitly introduced from the beginning. Rather. the quantum statistical nature of fermions prevents the two electrons from forming a tightly bound boson, and the boson from forming a cluster. This results in a repulsive force on the electrons and on the loosely bound pairs, which is a built in mechanism inherent in the system. These attractive and repulsive forces are properly balanced in BCS theory.

In the present theory, however, we explicitly take into account the correlation between the pairs in momentum space. This affects the balance between the attractive and repulsive forces, and results in a new balance between them. In addition to the direct-process $U$, Fig. 1(a), the pairing is further enhanced by the interaction between the pairs in momentum space, Fig. 1(b). The resulting system is more stable than the BCS system, illustrating a self-amplifying nature.

In Fig. 7, the strong $N(0)$ dependence of $F_{s}-F_{n}$ in the present system is compared with that in the BCS system. As discussed in Ref. 10, this suggests a cooperative nature of the pairing enhancement mechanism. Figure 7(a) 
( $T=0$ ) shows that the self-consistency mechanism of the present system differs widely from that of the BCS system characterized as the straight line in the same figure. However, in Fig. $7(\mathrm{~b})\left(T / T_{c}=0.4\right)$, the curve differs only slightly from the BCS system, indicating that, as the temperature is increased, the self-amplifying nature gradually disappears. Finally at $T=T_{c}$, the present system is reduced to the BCS system completely.

The self-amplifying nature of the present system appears in two different ways. At zero temperature, condensation is complete both in the BCS and the present system. As the temperature is increased, a fraction of electrons evaporate from the condensate. At low temperature, however, compared with the BCS system, the present system still possesses features of the complete condensate as shown in Figs. 5 and 6. In Fig. 5, the entropy is lower than that of the BCS system. In Fig. 6, as the temperature is increased, the energy increases more slowly than in the BCS system. Thus, compared with the BCS system at the same temperature, more electrons obey the pairing order, and the quasiparticle excitation is suppressed by the self-amplifying nature of the system.

However, as $T \rightarrow T_{c}$, the present system approaches the normal state more rapidly than does the BCS system, as illustrated by the rapid increase of the entropy and the energy just below $T_{c}$ in Figs. 5 and 6 , respectively. This rapid energy increase near $T_{c}$ suggests a large specific heat. The relative jump in the specific heat at $T_{c}, \Delta C / C_{n}$, can be used to estimate this rapid change quantitatively, because it does not depend on details of the system such as $N(0)$. In contrast to the quasiparticle excitation near $T=0$, the excitation just below $T_{c}$ is enhanced by the self-amplifying nature of the system. To study general properties near $T_{c}$, the Ginzburg-Landau expansion must be calculated, and this will be carried out in future work.

\section{B. Relationship to other studies}

First, in Ref. 9, Gyorffy, Staunton, and Stocks considered a way of going beyond the Gorkov decoupling by taking into account fluctuations around the Hartree-Fock potential. That is, they assumed the order parameter to be a random variable, and assigned a probability to it. They formulated the CPA condition as a fundamental equation of their treatment. This method corresponds to an approximation beyond the Gorkov decoupling. However, there is a possibility that, because the order parameter is assumed to be a static random variables, the possible state space which their solution occupies in the functional space is restricted. The relation between their conclusion and the present result is not clear.

Second, this paper does not directly examine the crossover problem between BCS-type pairs and bosonic tightly bound pairs. However, it is possible for the present scheme to provide us with a somewhat different point of view on this long-standing problem in attracting-fermion systems. In Refs. 21-25, two coupled equations, the BCS gap equation and the particle number equation, were formulated as a weak-coupling limit. From this limit, the system was extrapolated to the strong-coupling limit.
Therefore, only the type-A correlation in Sec. I was taken into account, and noninteracting composite bosons were obtained at the strong-coupling limit. If we expect interacting bosons at the strong-coupling limit, the type-B correlation in Sec. I must be included as well as the typeA correlation. In other words, instead of BCS superconductivity, superconductivity beyond the Gorkov decoupling should be assumed at the weak-coupling limit. This is an open problem.

\section{Relation to real materials}

As discussed in Sec. II, our model predicts the same transition temperature as that of the BCS model. Therefore, it is not appropriate for a theoretical explanation of the high- $T_{c}$ mechanism. However, compared with the condensate of the BCS system, that of the present system is more stable at low temperature. This fact provides another possibility. In some heavy-fermion superconductors such as $\mathrm{UBe}_{13}$, an anomalously large critical magnetic field $H_{c 2}(T=0)$ (approximately $100 \mathrm{kOe}$ ) has been reported. ${ }^{26}$ Considering the relatively low $T_{c}(<1 \mathrm{~K})$ of these materials, this $H_{c 2}(T=0)$ is unusual. There are two possibilities for it. The first is a large condensation energy $E_{s}-E_{n}$, and the second is a large GinzburgLandau parameter $(\kappa=\lambda / \xi)$. Hence the question arises whether these possibilities are explained naturally within the BCS theory and our scheme.

To explain the coexistence of large $E_{s}-E_{n}$ with low $T_{c}$ within the BCS scheme, a combination of high $N(0)$ and weak $U$ is necessary. Thus, for the heavy-fermion superconductors, anomalously high $N(0)$ must be assumed. As shown in Fig. 7, however, the present model has a far stronger dependence on $N(0)$ than does the BCS model. This enables us to explain anomalously large $H_{c 2}(T=0)$ values naturally, without anomalous high $N(0)$, which suggests that the present model is applicable to the heavy-fermion superconductors. As for the second possibility, compared with $\kappa$ in conventional type-II superconductors, an anomalously large $\kappa$ in heavy-fermion superconductors has not been reported. We plan to study the $\kappa$ of the present system in future work.

To compare the present theory with the heavy-fermion superconductors, it is also necessary to explain the large mass renormalization $(\delta m)$. In our theory, only the mean field that represents the correlation between the anomalous Green functions is considered in $\Omega_{3}$. As a result, in the superconducting phase the quasiparticles excited from the condensate become free particles, and in the normal phase the system becomes the ideal Fermi gas. To take into account the mass renormalization, a mean field $\Gamma_{n}$ must be included in $\Omega_{3}$ in addition to $\Gamma$, to represent the correlation between the quasiparticles. Using such a functional, all related quantities $\left(\delta m, \Gamma_{n}, \phi, \Gamma\right)$ must be determined self-consistently. If this is achieved, the correlation between the quasiparticles as well as that between the Cooper pairs is obtained. Accordingly, at $T=T_{c}$ the system undergoes a phase transition from the superconducting state to the nonideal Fermi gas rather than to the ideal Fermi gas as in the BCS and the present theory. This is an open problem. 
There are various aspects to be explored in heavyfermion systems. In this paper, we shed light on this complicated problem from the viewpoint of a selfconsistency mechanism.

\section{APPENDIX A}

From Eq. (1), the following derivative is obtained,

$$
\begin{aligned}
\frac{\partial \Omega_{1}}{\partial \phi^{*}(p)}= & \frac{1}{\beta}\left[\frac{\phi(p)}{\left(i \omega_{n}\right)^{2}-\varepsilon_{\mathrm{k}}^{2}-\left|\phi\left(i \omega_{n}\right)\right|^{2}}+F(p)\right] \\
& +\frac{1}{\beta} \frac{\partial F}{\partial \phi^{*}}\left[\phi^{*}(p)+U \frac{1}{\beta} \sum_{p^{\prime}} F^{*}\left(p^{\prime}\right)\right] \\
& +\frac{1}{\beta} \frac{\partial F^{*}}{\partial \phi^{*}}\left[\phi(p)+U \frac{1}{\beta} \sum_{p^{\prime}} F\left(p^{\prime}\right)\right],
\end{aligned}
$$

where $2 \phi^{*} F$ in Eq. (1) is rewritten as $\phi^{*} F+\phi F^{*}$. Using $\partial \Omega_{1} / \partial \phi^{*}=0$, the BCS gap equation

$$
\phi(p)=-U \frac{1}{\beta} \sum_{p^{\prime}} F\left(p^{\prime}\right)
$$

is obtained.

\section{APPENDIX B}

At zero temperature, Eq. (12) can be simplified, because the term that includes $g_{-}$vanishes. $g_{+}$is transformed as follows,

$$
\begin{aligned}
& \frac{\bar{\phi}^{2}}{E_{\mathrm{k}} E_{\mathrm{k}+\mathrm{q}}} \frac{1}{\omega-E_{\mathrm{k}}-E_{\mathrm{k}+\mathrm{q}}} \\
&=-\frac{\bar{\phi}^{2}}{E_{\mathrm{k}}} \int \frac{1}{E_{\mathrm{k}+\mathrm{q}}^{2}-\left(\omega-E_{\mathrm{k}}\right)^{2}} \\
&\left.\quad+\frac{\omega-E_{\mathrm{k}}}{E_{\mathrm{k}+\mathrm{q}}\left[E_{\mathrm{k}+\mathrm{q}}^{2}-\left(\omega-E_{\mathrm{k}}\right)^{2}\right]}\right) .
\end{aligned}
$$

Integrating the first and the second term with respect to $\varepsilon_{\mathbf{k}+\mathbf{q}}\left(=\varepsilon^{\prime}\right)$ results in $I_{s}^{(1)}\left(\varepsilon^{\prime}, \omega\right)$ and $\widetilde{\omega} I_{s}^{(2)}\left(\varepsilon^{\prime}, \omega\right)$, respectively.

At nonzero temperature, however, Eq. (12) cannot be simplified. The function in the integral of Eq. (12) is transformed in two ways. $g_{ \pm}$coupled to $f\left( \pm E_{\mathbf{k}}\right)$ in Eq. (12) is transformed as follows,

$$
\begin{aligned}
& \frac{\bar{\phi}^{2}}{E_{\mathbf{k}} E_{\mathbf{k}+\mathbf{q}}} \frac{1}{\omega-E_{\mathbf{k}} \mp E_{\mathbf{k}+\mathbf{q}}} \\
& \quad=\mp \frac{\bar{\phi}^{2}}{E_{\mathbf{k}}}\left(\frac{1}{E_{\mathbf{k}+\mathbf{q}}^{2}-\left(\omega-E_{\mathbf{k}}\right)^{2}}\right. \\
& \left.\quad \pm \frac{\omega-E_{\mathbf{k}}}{E_{\mathbf{k}+\mathbf{q}}\left[E_{\mathbf{k}+\mathbf{q}}^{2}-\left(\omega-E_{\mathbf{k}}\right)^{2}\right]}\right) .
\end{aligned}
$$

Integrating with respect to $\varepsilon_{\mathbf{k}+\mathbf{q}}\left(=\varepsilon^{\prime}\right)$ results in $I_{s}^{(1)}\left(\varepsilon^{\prime}, \omega\right)$ and $\widetilde{\omega} I_{s}^{(2)}\left(\varepsilon^{\prime}, \omega\right)$. On the other hand, $g_{ \pm}$coupled to $f\left( \pm E_{\mathbf{k}+\mathbf{q}}\right)$ in Eq. (12) is transformed as follows,

$$
\begin{aligned}
& \frac{\bar{\phi}^{2}}{E_{\mathrm{k}} E_{\mathrm{k}+\mathrm{q}}} \frac{1}{\omega-E_{\mathrm{k}} \mp E_{\mathrm{k}+\mathrm{q}}} \\
& =-\frac{\bar{\phi}^{2}}{E_{\mathrm{k}+\mathrm{q}}} \int \frac{1}{E_{\mathrm{k}}^{2}-\left(\omega \mp E_{\mathrm{k}+\mathrm{q}}\right)^{2}} \\
& \left.+\frac{\omega \mp E_{\mathbf{k}+\mathbf{q}}}{E_{\mathbf{k}}\left[E_{\mathbf{k}}^{2}-\left(\omega \mp E_{\mathbf{k}+\mathbf{q}}\right)^{2}\right]}\right) .
\end{aligned}
$$

Integrating with respect to $\varepsilon_{\mathrm{k}}(=\varepsilon)$ results in $I_{s}^{(1)}(\varepsilon, \omega)$ and $\widetilde{\omega} I_{s}^{(2)}(\varepsilon, \omega)$ for $g_{+}$, and $I_{s}^{\prime(1)}(\varepsilon, \omega)$ and $\widetilde{\omega} I_{s}^{\prime(2)}(\varepsilon, \omega)$ for $g-$.

A combination of these results leads to Eqs. (18)-(24).
${ }^{1}$ J. Bardeen, L. N. Cooper, and J. R. Schrieffer, Phys. Rev. 108, 1175 (1957).

${ }^{2}$ L. P. Gorkov, Zh. Eksp. Teor. Fiz. 34, 735 (1958) [Sov. Phys. JETP 7, 505 (1958)].

${ }^{3}$ G. R. Stewart, Rev. Mod. Phys. 56, 755 (1984); H. R. Ott, in Progress in Low Temperature Physics, XI (North-Holland, Amsterdam, 1987); U. Rauchschwalbe, Physica B + C 147, 1 (1987); Z. Fisk, D. Hess, C. Pethcik, D. Pines, J. Smith, J. Tompson, and T. Wills, Science 239, 33 (1988).

${ }^{4}$ T. Witt, Phys. Rev. Lett. 61, 1423 (1988).

5P. L. Gammel et al., Phys. Rev. Lett. 59, 2592 (1987); C. E. Gough et al; Nature 326, 855 (1987).

$6 \mathrm{~J}$. M. Blatt, Theory of Superconductivity (Academic, New York, 1964).

${ }^{7}$ There have been many studies on the superconductivity under many kinds of complex interactions which electrons experience in solids. In these studies, after renormalizing these interactions into the electron self-energy in many different ways, the conventional BCS self-consistency scheme is applied. These works proceed along a different line of thought from that of the present study.

${ }^{8}$ B. Giovannini, Helv. Phys. Acta. 50, 387 (1977).

${ }^{9}$ B. L. Gyorffy, J. B. Staunton, and G. M. Stocks, Phys. Rev. B 44, 5190 (1991).

${ }^{10}$ S. Koh, Physica C 191, 167 (1992).

${ }^{11}$ S. Koh, Nucl. Phys. A 560, 797 (1993). (Application here is to the atomic nucleus.)

${ }^{12}$ G. M. Eliashberg, Zh. Eksp. Teor. Fiz. 38, 966 (1960) [Sov. Phys. JETP 11, 696 (1960)].

${ }^{13}$ J. D. Scalapino, J. R. Schrieffer, and J. W. Wilkins, Phys. Rev. 148, 263 (1966).

${ }^{14}$ For a review, see J. P. Carbotte, Rev. Mod. Phys. 62, 1027 (1990).

${ }^{15}$ L. M. Luttinger and J. C. Ward, Phys. Rev. 118, 1418 (1960).

${ }^{16}$ G. M. Eliashberg, Zh. Eksp. Teor. Fiz. 43, 1005 (1962) [Sov. Phys. JETP 16, 780 (1963)]. 
${ }^{17}$ In Ref. 10, the symbol $G_{1}$ was used for $F$, and a negative sign in the definition of $F$ was left out. In the present paper, the conventional symbol $F$ is used and the sign is corrected.

18J. Bardeen and M. Stephen, Phys. Rev. 136A, 1485 (1964).

${ }^{19}$ Y. Wada, Phys. Rev. 135, A1481 (1964).

${ }^{20}$ In Ref. 10, the expression for $\Gamma(\omega)$ was obtained at zero temperature. However, in Eqs. (11) and (12) of that paper, a factor $\frac{1}{2}$ before the integral was let out, and it must be corrected. Furthermore, in that paper, a positive $U^{\prime}(=-U)$ and $\Gamma^{\prime}(=-\Gamma)$ were redefined in Sec. III. In the present paper, to avoid confusion, we use the same sign of $U$ and $\Gamma$ throughout the paper.
${ }^{21}$ A. J. Leggett, in Modern Trends in the Theory of Condensed Matter (Springer, Berlin, 1989), p. 14.

${ }^{22}$ P. Nozières and S. Schmitt-Rink, J. Low. Temp. Phys. 59, 195 (1985).

${ }^{23}$ M. Randeria, J. M. Duan, and L. Y. Shieh, Phys. Rev. B 41, 327 (1990).

${ }^{24}$ D. van der Marel, Physica C 135, 35 (1990).

${ }^{25}$ A. Tokumitsu, K. Miyake, and K. Yamada, J. Phys. Soc. Jpn. 60, 380 (1991).

${ }^{26}$ M. B. Maple, J. W. Chen, S. E. Lambert, Z. Fisk, J. L. Smith, H. R. Ott, J. S. Brooks, and M. J. Naughton, Phys. Rev. Lett. 54, 477 (1985). 